
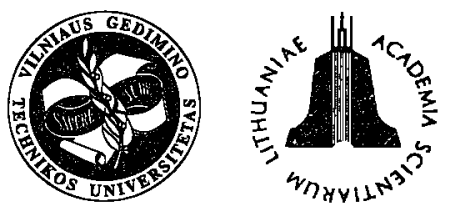

ISSN 1648-4142 TRANSPORT

http:/www.vtu.1t/english/editions

TRANSPORT - 2003, Vol XVIII, No 2, 61-65

\title{
EFFICIENCY ESTIMATION OF TRANSPORT COMPANIES' ACTIVITY
}

\author{
Andrius Vyskupaitis \\ Dept of Transport Management, Vilnius Gediminas Technical University, \\ Plytines g. 27,LT-2016 Lithuania. E-mail: a.vyskupaitis@centras.lt
}

Received 20021015 ; accepted 20030228

\begin{abstract}
In this article methodological subjects of activities efficiency research of the transport companies as well as other complex systems are analyzed. Methodically it is the most important to select estimation indexes of efficiency of searching object activities. After the system of indexes is formed, it is determined that it is more useful to use the system of technical economic indexes in order to estimate the level of efficiency of the activities of transport companies. It is so, because none of the separate indexes individually is able comprehensively to reflect the activities of transport systems or separate companies. Using the proposed method it is possible to research the great amount of objects according to numerous indexes. The main task is to form conditionally monolithic groups from the totality of objects. The level of activities of the objects in these groups should be similar. It would be possible to solve this task with the help of the image identification theory. The essence of this theory is to used necessary algorithm for the identification of searching objects signs. On this basis each object is reckoned in a certain monolithic objects group. Next step is the use of classification algorithms, which enable to classify the whole of searching objects. In this article you would find two main stages of classification problem solution. In the first stage the expansion of the objects into monolithic groups according to one of the indexes goes. The quality of this stage depends on the amount of the groups and on the selection of a concrete index. In the second stage the final objects expansion into monolithic groups according to the whole of selected technicaleconomic level indexes goes. Here is the manifold classification of the objects. Its results enable to determine the efficiency level of transport systems activities as well as to improve the quality of planning transport company's activities.

Keywords: transport; efficiency; planning; image identification theory; classification; methods.
\end{abstract}

\section{Introduction}

The article gives the analysis of the topical problem of transport companies related to the improvement of the activity planning. The aim of the research is to offer a new technique for estimating the efficiency of transport companies, as well as to improve the quality of the activity planning thereof. One of the most important tasks in solving this problem is selecting of the estimation index system. The article presents and describes the main methodological principles on the formation of such system of estimation indexes. The presentation is followed by the detailed analysis of the possibility to use a new method for solving the tasks of transport. A new technique is offered for solving of the classification tasks on the basis of the image identification theory.

This method enables to objectively estimate the efficiency level achieved by the transport companies. This gives the possibility to perform the qualitative analysis of the transport companies and creates the basis for the activity planning thereof [1].

\section{Formation Methods of the Estimation Index System}

The growth of computerisation in all fields of economy made it possible to process vast amounts of information by creating background for making the right decisions in planning and controlling the operation of complex systems, including those of transport companies. On the other hand, the rise of these possibilities sets new qualitative requirements related with the improvement of the economic efficiency research methods in transport systems.

Methodologically, in order to objectively and precisely estimate the efficiency level of a certain transport system or a separate company the most complicated problem remains selecting of the estimation index system [2]. It has taken time to find such complex, resumptive indexes (e.g., transport operation scope in ton kilometres or passenger kilometres, etc.) or an entire index system. The importance of resumptive indexes was great before, two or three decades ago, when the computerisation level of systems and processes control was still quite low and the possibilities of processing large information flows were limited.

New economic-mathematical methods appeared and the improvement of the technical base made it possible to process huge amount of initial information for more objective estimation of the efficiency level of entities and 
for preparing the decision-making in controlling and planning.

The application of the economic index system in estimating the efficiency level of transport companies is purposeful also because no complex, resumptive indexes, no matter how important they may be, can individually provide a versatile and complete view of the activity of transport systems or separate companies. Therefore, we will now try to ground the new method for estimating the efficiency level of separate transport companies in Lithuania (or larger regions) and to compare the economic activity level achieved by these companies.

Besides, the results received using this method create the base for improving the activity planning of transport companies.

As the experience shows, for certain companies it is useful to compare the conditions of their activity and the economic results not only basing on the general economic indexes, but also on the total factors determining the changes thereof [3]. Then two possibilities appear: first to compare the activity of two separate transport companies and to estimate the results in hard restricting conditions (i.e. actual activity conditions of the companies), second to do the same estimating the possible variations of the activity conditions, which is very important in the planning practice.

Another important problem faced by the economists in estimating the technical-economic level of the activity results of various transport companies or systems is selecting of principles for comparing the activity results of separate objects.

Two variants are possible here:

1) the issue of comparing two separate indexes of the companies' activity is solved, and then the groups are formed on the basis of the calculated partial comparable indexes and group comparable indexes are calculated;

2) from all of the analysed companies the most monolithic groups thereof are formed, and after that the technical-economic level of these groups is compared on the basis of the average index values.

The first variant is only possible with a small number of objects and an insignificant number of indexes.

The second variant is a much more promising one the results are compared on the basis of the most monolithic object groups.

To achieve the set objectives we suggest applying the method of comparative analysis to analyse the economic results of an unlimited number of transport companies. For this purpose we need to form relatively monolithic groups of companies according to the technical-economic level achieved by the companies. The method is implemented on the basis of the block scheme (Fig).

The methodical views are based on the following principles reflecting general features and differences of the transport companies:

1. To reduce the amount of the research not separate transport companies are analysed, but monolithic groups

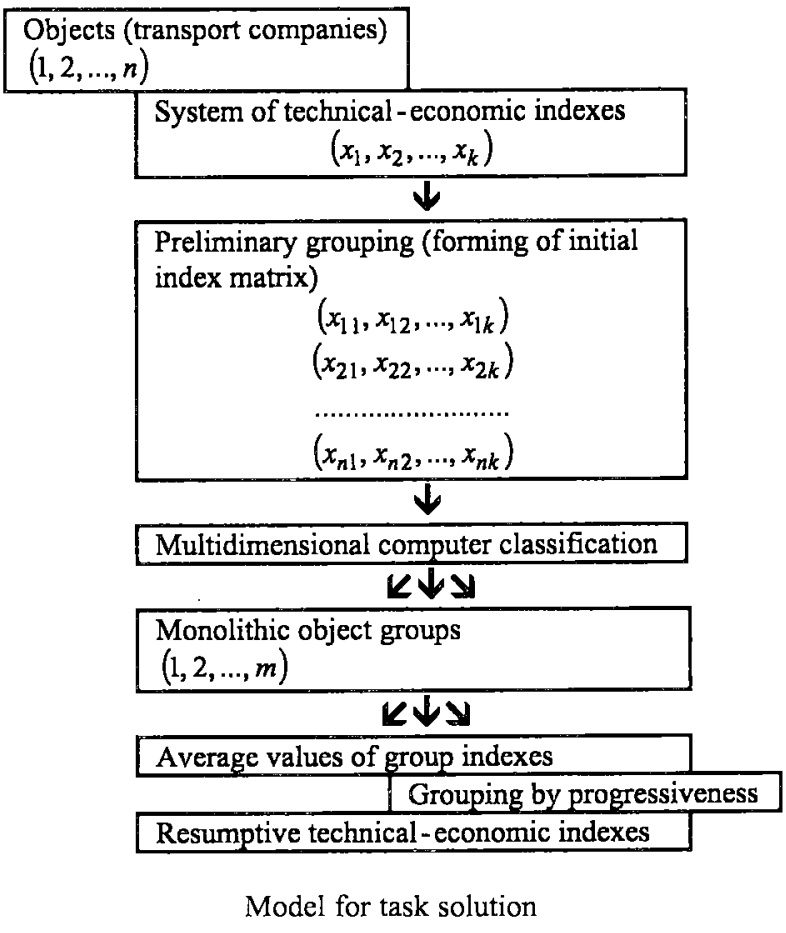

thereof are formed (according to the selected system of technical-economic indexes).

2. Common technological and organisational transport operation is characteristic of all transport companies (separately carrying passengers and goods).

3 . In the process of the activity the companies proceed under relatively similar conditions (construction of buildings, acquiring of vehicles and other machinery, parking renovation, reconstruction, development, etc.).

4. The system of technical-economic indexes is the same for transport companies and their groups, and the calculation methods are united.

5. The resources used in the process of work are the same.

6. Variation of each of the technical-economic index is of the stochastic character, therefore it is possible to use united methods to estimate the changes.

It follows that the most important task is forming relatively monolithic groups of many transport companies. The formation of such groups is necessary for several reasons.

First - transport companies are not equally developed, they have different working experience, they belong to different generations. Typical features of the production and activity process are characteristic of each stage of the companies' development (age and structure of the park, technique and technology level, transport organising, etc.). Companies established at the same or near period usually are on the similar technique and technology level and they are at the same points of their development path [4].

Companies of different "generations", on the contrary, quite much differ in their technical-economic characteristics. 
Second - objects of the same "generation" may in their turn differ in the achieved technical-economic level and are classified in to different groups. Here the principle of the companies' monolithic character might be their production capacities. Parking characteristics of large and small companies are different; they also differ in transport organising.

The fact that a certain variety is characteristic of each group of companies from the point of view of space and time makes us search for the ways to divide the companies into more or less monolithic groups in a reliable way [5].

Third - the objective of the analysis of the whole transport system operation and the estimation of the achieved level and development tendencies is to give the picture not only of the transport system development in the whole country, but also of individual elements thereof (transport companies and their groups).

\section{Use of the Image Identification Theory to Solve the Classification Tasks}

The economists are constantly searching for more effective ways of solving the classification tasks. In my opinion, the set tasks today can be solved on the basis of the image identification theory [6]. The general point of the image identification theory is as follows:

We have a certain object of study, e.g. the totality of transport companies. The appropriate information available about the aforementioned objects allows us to divide the totality into separate groups using the corresponding classification criterion. At the same time we have to form the so-called list of features enabling us to describe each group of objects separately. In our case dividing the totality of transport companies into monolithic groups, such list of features consists of the system of technical-economic indexes as described by the block scheme $\left(x_{1}, x_{2}, \ldots, x_{k}\right)$.

Further it is necessary to use the algorithm enabling to identify the features of the objects. Functioning of the identification system enables checking of the unknown objects by testing with the help of certain measures on the basis of which the features of this object are determined. Then on the basis of the established features of the unknown object it is determined by identification algorithms as to what group the analysed objects should be ascribed. The experience of creating the identification systems shows that practical implementation of each identification system, as a rule, is related to the solution of tasks complex. Each of these tasks is an integral part of the whole image identification system.

On the basis of the image identification theory we suggest the method for estimating the results of transport companies' activity, which is implemented in the following stages:

1. Task formulation and describing of the conditions for the task solution;

2. Economic analysis of the studied objects;
3. Determining of the state and structure of the initial information;

4. Collecting of the initial information and its initial processing;

5. Statistic processing of the initial information;

6. Computer processing of the information by the respective algorithm;

7. Interpretation of the computer processed results;

8. Determining of the reliability and stability of the obtained results;

9. Recommendations on the use of the obtained results improving the planning of transport companies' activity.

While creating the identification systems the most important thing is to ensure the conditions enabling to analyse the identified objects as detailed and properly as possible. The aim of this stage is to determine what are the qualities of the analysed objects, what joins or separates them. The analysis of the identified objects gives objective assumptions to successful solving of the following identification task, i.e. to classify the totality of the objects. The choice of the classification principles is determined by the requirements for the identification system, which in their turn depend on what conclusions and decisions can be made having evaluated the identification of the "unknown" objects' system.

It is obvious that the solution of these tasks is first of all conditioned by the essence of the analysed phenomena and the aims that are set for the research before it is started. In our case such aims are the following:

- Dividing the totality of the transport companies in Lithuania into monolithic object groups basing on the indexes characterising the technical-economic level of the companies' activity;

- Estimating "progressiveness" of the obtained groups;

- Comparing the technical-economic level of the obtained object groups;

- Further planning of the company activity estimating the results of the research.

The attention will be focused on search for the methods to divide the objects into monolithic groups, which (the methods) are adequate to the particularity of the task to be solved.

They have been approved in many fields of production, however they have not yet been applied in road transport. The analysis has shown that this method has advantages and at the same time is quite simple as compared with other methods applied to solve similar economic tasks. The classification algorithm used by us especially suits to identify the objects with characteristic features expressed by numbers [7].

According to the suggested classification algorithm, segmentation $A$ is more advantageous than segmentation $B$, provided that the distance between two nearest object groups in segmentation $A$ is larger than that between the object groups in segmentation $B$. Formally this means: 
$A$ is better than $B$ provided that:

$$
Q_{i j}^{A}=\max Q_{i j}^{A}<\max Q_{t s}^{B}=Q_{t s}^{B},
$$

where: $i, j$-group numbers in segmentation $A ; \mathrm{t}, \mathrm{s}-$ group numbers in segmentation $B ; Q_{i j}$ - distance index between groups $i$ and $j$.

Besides, the group data is the more distant the lower is the value of $Q$. If $Q^{A}=Q^{B}$ (situation when in both segmentations the closest groups are formed from the same points), then we have to analyse the next closest pair of groups in each segmentation and compare them.

The essence of the selected algorithm is the segmentation of the objects into groups, which corresponds to the local minimum of the segmentation criterion. Besides, as the initial condition a certain preliminary grouping of the objects must be done on the basis of one of the indexes from the dictionary of features $\left(x_{1}, x_{2}, \ldots, x_{k}\right)$.

The quality of the preliminary segmentation depends on the number of the selected groups and on the index according to which the preliminary segmentation of the objects is carried out.

The number of groups is determined by the way of expertise depending on the number of the analysed objects and on the unevenness of the index according to which the preliminary segmentation of the objects is carried out in the value distribution space (majority of the groups are preconditioned by the requirement that the groups should contain a sufficient number of objects).

To carry out the preliminary segmentation of the objects the analysis algorithm is suggested according to which the segmentation is carried out in the following order:

Provided that $\max \left(x_{l}\right)$ and $\min \left(x_{l}\right)$ are the maximum and minimum values of the variable $l$ According to which the preliminary segmentation of the objects in to groups is carried out. The class diameter is determined:

$$
C=\frac{\max \left(x_{l}\right)-\min \left(x_{l}\right)}{M},
$$

where $M$ - number of classes determined by the researcher.

Then the class limits are determined by the following formula:

$$
C_{k, k+1}=\min \left(x_{l}\right)+C \cdot k ;(k=1,2, \ldots, M-1) .
$$

All we have to do now is to properly prepare the arrays of the preliminary data. The index system of the technological-economic level of all analysed transport companies must be the same, i.e. the same number of the same indexes for the same (or several) year.

The algorithm provides for the possibility to vary the number of technical-economic indexes according to which the final segmentation of the objects by number will be carried out. The researchers can also change the number of the objects and the composition of the groups (changing the index according to which the initial grouping of the companies is carried out). After preliminary segmentation of the objects into groups is done, the next step is to "transfer" the results of preliminary segmentation to the algorithm "Improved segmentation". Adopting of this algorithm enables to purposively recheck the closest object groups varying their number and composition in order to achieve optimal segmentation into monolithic groups. When a pair of groups with minimal distance is checked, the points are determined therein closest to another group. In case when transferring a specific index to another group at least one of the variants improves the segmentation quality, the result is "memorised" and the whole procedure is restarted in order to further improve the segmentation quality.

If in none of the cases the quality of the segmentation criterion is improved, then the next closest pair of groups is checked. The procedure is repeated with this pair transferring the points into the next group. The calculation is finished if the segmentation quality criterion cannot be improved for any of the group pairs. This means that the minimum value $Q$ of the segmentation criterion is achieved.

Selecting different variants of the preliminary segmentation which are more or less clear to the experts it is possible to achieve different local minimums, which are later compared by the same criterion. Actually, after the described "checking" the segmentation quality is improved by $1,5-2$ times as compared with the preliminary segmentation.

The next stage is the final grouping of the objects by the totality of the selected technological-economic index level, i.e. the algorithm is used enabling implementation of the multidimensional classification of objects which is hard to imagine in space. This results in the final composition and structure of the monolithic object groups taking into consideration all the factors that have influenced the level of all analysed transport companies in a certain year.

Possible application of this method for the estimation of the achieved technical-economic level becomes much wider when the estimation tendencies are clarified in time. When determining the monolithic object groups by the aforementioned method it is possible to define the tendencies for the objects to be transferred from one group into another in each of the several recent years and, what is most important, the reasons for these tendencies. The estimation of these reasons would significantly improve the quality of planning the activity of transport companies.

\section{Conclusions}

1. To estimate the efficiency of transport companies we have to use not separate efficiency indexes but the whole system of indexes. In this way technical-economic level of the companies' efficiency is reflected more objectively and universally.

2. The suggested method enables to analyse the indefinite number of objects estimating tens and hundreds of their activity indexes. 
3. The article grounds the new approach towards the efficiency estimation of the analysed transport companies' activity by grouping the companies into relatively monolithic groups.

4. With large arrays of preliminary data it is recommended to solve the classification tasks on the basis of the image identification theory using multidimensional classification algorithms.

5. The results of the analysis give significantly more objective estimation of the efficiency level achieved by the companies and enable to improve the quality of planning their activity.

\section{References}

1. Kotler, P. Marketing Management. Analysis, Planning, Implementation and Control. Prentice Hall International Inc. 1991. $756 \mathrm{p}$.

2. Hallmark, S. L.; Guensler, R. and Fomunung I.
Charakterizing on-road variables that affect passenger vehicle modal operation. Transportation research part D-transport and environment, Vol 7D, No 2, 2002, p. 81-98.

3. Baublys, A. The introduction to Transport System Theory (Transporto sistemos teorijos ivadas). Vilnius: Technika, 1997. 298 p. (in Lithuanian).

4. Baublys, A. Assessment of statistical probability of the technological transportation process. Transport, Vol XVII, No 4, 2002, p. 127-136.

5. Goulias, K. G. Multilevel analysis of daily time use and time allocation to activity types accounting for complex covariance structures using correlated random effects. Transportation, Vol 29, No 1, 2002, p. 31-48.

6. Rozin, B. B. Image identification theory in economic research (Теория распознавания образов в экономических исследованиях). Moscow: Statistika, 1973 (in Russian).

7. Aivazian, S. M. and i. e. The classification of multidimentional observations (Классификация многомерных наблюдений). Moscow: Statistika, 1974 (in Russian). 